\title{
Multispin Giant Magnons
}

\author{
N.P. Bobev ${ }^{\star}$ and R.C. Rashkov ${ }^{\dagger 1}$ \\ * Department of Physics and Astronomy, University of Southern California, Los Angeles, CA \\ 90089-0484, USA \\ ${ }^{\dagger}$ Department of Physics, Sofia University, 1164 Sofia, Bulgaria
}

\begin{abstract}
We investigate giant magnons from classical rotating strings in two different backgrounds. First we generalize the solution of Hofman and Maldacena and investigate new magnon excitations of a spin chain which are dual to a string on $R \times S^{5}$ with two nonvanishing angular momenta. Allowing string dynamics along the third angle in the five sphere, we find a dispersion relation that reproduces the Hofman and Maldacena one and the one found by Dorey for the two spin case. In the second part of the paper we generalize the two "spin" giant magnon to the case of $\beta$-deformed $A d S_{5} \times S^{5}$ background. We find agreement between the dispersion relation of the rotating string and the proposed dispersion relation of the magnon bound state on the spin chain.
\end{abstract}

\footnotetext{
${ }^{1}$ e-mail: rash@phys.uni-sofia.bg; bobev@usc.edu
} 


\section{Introduction}

The AdS/CFT correspondence [1] is a powerful tool for studies and future advances in the understanding of gauge theories at strong coupling. One of the predictions of the correspondence is the equivalence between the spectrum of free string theory on $A d S_{5} \times S^{5}$ and the spectrum of anomalous dimensions of gauge invariant operators in the planar $\mathcal{N}=4$ Supersymmetric YangMills (SYM) theory. In order to check this conjecture we need the full spectrum of free string theory on a curved space, such as $A d S_{5} \times S^{5}$, which is still an unsolved problem. However in certain limits the problem becomes tractable and some comparisons on both side of the correspondence can be made. It turns out that the problem simplifies in the limit of string states with large quantum numbers [2],[3] and one can use a semiclassical approximation on the string side to find the energy spectrum. In order to test the predictions of the correspondence a way to compute the anomalous dimensions of long single trace operators (dual to the strings with large quantum numbers) on the gauge theory side was needed. Minahan and Zarembo proposed a remarkable solution to this problem [12] by relating the Hamiltonian of the Heisenberg spin chain with the dilatation operator of $\mathcal{N}=4 \mathrm{SYM}$. On other hand, in several papers the relation between strings and spin chains was established, see for instance [16],[17],[18],[19] and references therein. This idea opened the way for a remarkable interplay between spin chains, gauge theories, string theory ${ }^{2}$ and integrability (the integrability of classical strings on $A d S_{5} \times S^{5}$ was proven in [21]).

Having the above relations, it was natural to look for string solutions governing various corners of the spin chain spectrum. The most studied cases were spin waves in long-wave approximation corresponding to rotating and pulsating strings in certain limits, see for instance reviews $[13],[14],[15]$ and references therein. Another interesting case are the low lying spin chain states corresponding to the magnon excitations. One class of string solutions already presented in a number of papers is the string theory on pp-wave backgrounds. The later, although interesting and important, describe point-like strings which are only part of the whole picture. The question of more general string solutions corresponding to this part of the spectrum was still unsolved.

Recently Maldacena and Hofman[4] were able to map spin chain "magnon" states to specific rotating semiclassical string states on $R \times S^{2}$. This result was soon generalized to magnon bound states ([5],[6],[7],[8]), dual to strings on $R \times S^{3}$ with two non-vanishing angular momenta. Moreover in [8] a different giant magnon state with two spins was found, it is dual to string

\footnotetext{
${ }^{2}$ For very nice reviews on the subject with a complete list of references see [13],[14],[15]
} 
moving on $A d S_{3} \times S^{1}$ i.e. it has spin in both the AdS and the spherical part of the background. It is natural to generalize this construction and look for giant magnons with multiple spins, these should be dual to rotating strings on $R \times S^{5}$ with 3 spins.

The relation between energy and angular momentum for the one spin giant magnon found in [4] is:

$$
E-J=\frac{\sqrt{\lambda}}{\pi}\left|\sin \frac{p}{2}\right|
$$

where $p$ is the magnon momentum which on the string side is interpreted as a difference in the angle $\phi$ (see [4] for details). In the two spin case the $E-J$ relation was found both on the string $[6],[7],[8]$ and spin chain [5] sides and looks like:

$$
E-J=\sqrt{J_{2}^{2}+\frac{\lambda}{\pi^{2}} \sin ^{2} \frac{p}{2}}
$$

where $J_{2}$ is the second spin of the string. Allowing analogous string dynamics on the third angle of the five sphere one can ask what is the dispersion relation and to which magnon excitations it should correspond. For for the particular string configuration we consider in the first part of the paper we find agreement with two spin magnon excitations on the spin chain side ${ }^{3}$.

In the second part of the paper we will investigate multi spin giant magnons in the $\beta$-deformed $A d S_{5} \times S^{5}$ background found by Lunin and Maldacena [22] ${ }^{4}$. We will generalize the results in [9] by considering a more general rotating string anzatz. The same anzatz was considered in [7],[8] for the pure $A d S_{5} \times S^{5}$ case. It was argued in [5] that this simple rotating string with two angular momenta corresponds to a bound state of magnons propagating on the spin chain. The second angular momentum $J_{2}$ was interpreted as the number of magnons in this bound state (this number can be finite). As expected in the limit of vanishing deformation - $\beta \rightarrow 0$ we reproduce the dispersion relation from the pure $A d S_{5} \times S^{5}$ case.

The paper is organized as follows: In section 2 we briefly describe the Hofman-Maldacena giant magnon solution from strings on $R \times S^{2}$ and its recently found two spin generalization. We then proceed with applying the same idea to rotating strings on $R \times S^{5}$ and find the energymomentum relation for these strings. In section three we turn our attention to the so called $\beta$-deformed $A d S_{5} \times S^{5}$ background and investigate two spin giant magnons in it. This is a generalization of the two spin giant magnon presented in section 2 and is different from the

\footnotetext{
${ }^{3}$ We thank A.A. Tseytlin for comments on this point

${ }^{4}$ For related work on string/gauge/spin chain duality in $\beta$-deformed backgrounds see [23]-[40]
} 
solution found in [9]. In the last section we present our conclusions and a few possible directions for further studies.

\section{Giant magnons from strings on $A d S_{5} \times S^{5}$}

Here we will review the idea of Hofman and Maldacena [4] for the stringy description of spin chain magnon excitations. Let us look of a special class of gauge invariant operators which are characterized by an infinite value of one of the $S O(6)$ charges $J$ and have finite value of $E-J$. When $E-J$ vanishes the operator is composed of a chain of $J$ operators, namely:

$$
\mathcal{O} \sim \operatorname{Tr}(Z Z . . Z \ldots Z)
$$

On this "chain" of we can consider a finite number of fields $Y$ which can be interpreted as excitations propagating on the chain. The corresponding operator takes the form:

$$
\mathcal{O} \sim \sum_{k} e^{i k p}(Z . . Z Z Y Z Z \ldots Z)
$$

Where the summation is over all possible positions on the chain where $Y$ can be inserted. This picture on the gauge side has a description in terms of spin chains [12]. The operators $Z$ represent the ground state of the chain and the $Y$ s correspond to magnon excitations.

Using supersymmetry arguments Beisert [10] was able to find the following dispersion relation of the magnon excitations:

$$
E-J=\sqrt{1+\frac{\lambda}{\pi^{2}} \sin ^{2} \frac{p}{2}}
$$

Here $p$ is the magnon momentum and the periodicity in $p$ is due to the discreteness of the spin chain. The large 't Hooft coupling limit of the above relation is:

$$
E-J=\frac{\sqrt{\lambda}}{\pi}\left|\sin \frac{p}{2}\right|
$$

Hofman and Maldacena were able to reproduce the above relation from string theory on a $R \times S^{2}$ subspace of $A d S_{5} \times S^{5}$. They considered a simple string with both ends rotating on the equator of $S^{2}$ with constant separation between the ends $\Delta \varphi$. The crucial step was to identify 
this angle difference with the momentum of the spin chain magnon $\Delta \varphi=p$. In order to be able to reproduce exactly the magnon dispersion relation the following limits are considered:

$$
J \rightarrow \infty \quad E-J=\text { fixed } \quad \lambda=\text { fixed } \quad p=\text { fixed }
$$

We note that a closed folded string configuration with one angular momentum as the one considered in [3] will correspond to a state which is a superposition of two magnons with opposite momenta. The solution we are interested in lives on the $R \times S^{2}$ background with the metric:

$$
d s^{2}=-d t^{2}+d \theta^{2}+\sin ^{2} \theta d \varphi^{2}
$$

We look for a rotating string solution of the Polyakov action of this model of the form:

$$
t=\tau \quad \theta=\theta(y) \quad \varphi=t+\tilde{\varphi}(y)
$$

Where we have defined the variable $y=c x-d t$. Here $x$ is a world sheet spatial coordinate with infinite range and $c$ and $d$ are constants. Using the above anzatz it is not hard to integrate the equations of motion and get:

$$
\cos \theta=\frac{\cos \theta_{0}}{\cosh y} \quad \tan \tilde{\varphi}=\frac{\tanh y}{\tan \theta_{0}}
$$

The constants $c$ and $d$ are related to the integration constant $\theta_{0}$ by:

$$
\frac{1}{c}=\cos \theta_{0} \quad d=\tan \theta_{0}
$$

Now it is easy to see that $c^{2}-d^{2}=1$, this provides us with the necessary condition that the group velocity $v=\frac{d}{c}$ is less than one. We are interested in the conserved charges for our system:

$$
E=\frac{\sqrt{\lambda}}{2 \pi} \int_{-\infty}^{\infty} d x \quad J=\frac{\sqrt{\lambda}}{2 \pi} \int_{-\infty}^{\infty} d x \sin ^{2} \theta
$$

Using the above relation we see that

$$
E-J=\frac{\sqrt{\lambda}}{\pi} \cos \theta_{0}
$$


Now the proposal of Hofman and Maldacena [4] is to make the identification $\cos \theta_{0}=\sin (p / 2)$. This leads to the conclusion that the dispersion relations of the magnon excitation of the infinte spin chain in the large 't Hooft coupling limit and the rotating string with one large angular momentum are the same. Thus we have outlined the idea in [4] for finding the string dual description of a giant magnon excitation in a certain limit. This opens the possibility for generalizing this construction and studying multi magnon bound states, which are argued to be dual [5] to rotating strings with two angular momenta. We proceed with this analysis in the following sections.

\subsection{Two spin giant magnons in $A d S_{5} \times S^{5}$}

Here we will present the two spin rotating string solution found in [7] in a slightly different notation which will be more convenient when we analyze the $\gamma$-deformed background. This rotating string is dual to a bound state of $J_{2}$ magnons with charge $J_{1}$. We look for classical string solution on a $R \times S^{3}$ subspace of $A d S_{5} \times S^{5}$ :

$$
d s^{2}=-d t^{2}+d \theta^{2}+\sin ^{2} \theta d \phi_{1}^{2}+\cos ^{2} \theta d \phi_{2}^{2}
$$

The Polyakov action for this system is:

$S_{P}=\frac{\sqrt{\lambda}}{4 \pi} \int d \tau d \sigma\left[-\left(\partial_{\tau} t\right)^{2}-\left(\partial_{\tau} \theta\right)^{2}+\left(\partial_{\sigma} \theta\right)^{2}+\sin ^{2} \theta\left(\left(\partial_{\sigma} \phi_{1}\right)^{2}-\left(\partial_{\tau} \phi_{1}\right)^{2}\right)+\cos ^{2} \theta\left(\left(\partial_{\sigma} \phi_{2}\right)^{2}-\left(\partial_{\tau} \phi_{2}\right)^{2}\right)\right]$

It can be checked that the following anzatz is consistent with the equations of motion following from the above action and the Virasoro constraints:

$$
\theta=\theta(y) \quad \phi_{1}=t+g_{1}(y) \quad \phi_{2}=\nu t+g_{2}(y)
$$

Here we have defined a new variable $y=c \sigma-d \tau$ and we work in the conformal gauge $t=\tau$. It is easy to derive the following equations for the unknown functions $g_{1}(y), g_{2}(y)$ and $\theta(y)$ :

$$
\begin{aligned}
& \partial_{y} g_{1}=\frac{d}{\sin ^{2} \theta}-d \quad \partial_{y} g_{2}=-\nu d \\
& \partial_{y} \theta=\cos \theta \sqrt{1-\nu^{2} c^{2}-d^{2} \frac{\cos ^{2} \theta}{\sin ^{2} \theta}}
\end{aligned}
$$


We can integrate the last equation and find $\cos \theta$ to be:

$$
\cos \theta=\sqrt{\frac{1-\nu^{2} c^{2}}{c^{2}-\nu^{2} c^{2}}} \frac{1}{\cosh \left(\sqrt{1-\nu^{2} c^{2}} y\right)}
$$

We denote

$$
\cos \theta_{0}=\sqrt{\frac{1-\nu^{2} c^{2}}{c^{2}-\nu^{2} c^{2}}}
$$

and following [4] we identify $\sin \frac{p}{2}$ with $\cos \theta_{0}$, where $p$ is the momentum of the magnon (interpreted as a geometrical angle in the string picture). Knowing the solution $\theta(y)$ we can find explicitly the conserved charges of the rotating string:

$$
\begin{aligned}
& E=\frac{\sqrt{\lambda}}{2 \pi} \int_{-\infty}^{\infty} d \sigma \\
& J_{1}=\frac{\sqrt{\lambda}}{2 \pi} \int_{-\infty}^{\infty} d \sigma \sin ^{2} \theta\left(1-d \partial_{y} g_{1}\right) \\
& J_{2}=\frac{\sqrt{\lambda}}{2 \pi} \int_{-\infty}^{\infty} d \sigma \cos ^{2} \theta\left(\nu-d \partial_{y} g_{2}\right)
\end{aligned}
$$

After a little algebra we arrive at:

$$
E-J_{1}=\frac{J_{2}}{\nu}=\frac{\sqrt{\lambda}}{\pi} \frac{c \cos ^{2} \theta_{0}}{\sqrt{1-\nu^{2} c^{2}}}
$$

Finally we extract the dispersion relation for the magnon bound state found in [5] and derived from the string sigma model on $R \times S^{3}$ in [7],[8]:

$$
E-J_{1}=\sqrt{J_{2}^{2}+\frac{\lambda}{\pi^{2}} \sin ^{2} \frac{p}{2}}
$$

\subsection{More two spin giant magnons on $R \times S^{5}$}

Now we proceed to the generalization of the ideas mentioned above by looking at a more general ansatz allowing string dynamics on three angles in $S^{5}$ part of the geometry. In this section we will closely follow the notation of [7] which we find more suitable for our ansatz than the method 
based on the Complex sine-Gordon model used for the analysis of the two spin case in [6]. The background of interest is ${ }^{5}$ :

$$
d s^{2}=-d t^{2}+d \theta^{2}+\cos ^{2} \theta d \phi_{3}^{2}+\sin ^{2} \theta\left(d \psi^{2}+\cos ^{2} \psi d \phi_{1}^{2}+\sin ^{2} \psi d \phi_{2}^{2}\right)
$$

Now we will define a new coordinate $z=\sin \theta$, fix $\psi=\pi / 4$ and work in conformal gauge $t=\tau$. The Polyakov action takes the form:

$$
\begin{aligned}
& S_{P}=-\frac{\sqrt{\lambda}}{4 \pi} \int d \sigma d \tau\left[-\dot{t}^{2}+\frac{1}{1-z^{2}}\left(\left(\partial_{\sigma} z\right)^{2}-\left(\partial_{\tau} z\right)^{2}\right)+\left(1-z^{2}\right)\left(\left(\partial_{\sigma} \phi_{3}\right)^{2}-\left(\partial_{\tau} \phi_{3}\right)^{2}\right)\right. \\
& \left.\frac{z^{2}}{2}\left(\left(\partial_{\sigma} \phi_{1}\right)^{2}-\left(\partial_{\tau} \phi_{1}\right)^{2}+\left(\partial_{\sigma} \phi_{2}\right)^{2}-\left(\partial_{\tau} \phi_{2}\right)^{2}\right)\right]
\end{aligned}
$$

We should impose also the Virasoro constraints:

$$
\begin{aligned}
& \frac{\dot{z} z^{\prime}}{1-z^{2}}+\left(1-z^{2}\right) \dot{\phi}_{3} \phi_{3}^{\prime}+z^{2}\left(\dot{\phi}_{1} \phi_{1}^{\prime}+\dot{\phi}_{2} \phi_{2}^{\prime}\right)=0 \\
& \frac{\dot{z}^{2}+z^{\prime 2}}{1-z^{2}}+\left(1-z^{2}\right)\left(\dot{\phi}_{3}{ }^{2}+\phi_{3}^{\prime 2}\right)+z^{2}\left(\dot{\phi}_{1}{ }^{2}+\phi_{1}^{\prime 2}+\dot{\phi}_{1}{ }^{2}+\phi_{2}^{\prime 2}\right)=1
\end{aligned}
$$

It can be shown that the following anzatz is consistent with the equations of motion and the above constraints:

$$
z=z(\sigma-v \tau) \quad \phi=\tau+g(\sigma-v \tau) \quad \phi_{1}=\omega_{1} \tau-v \omega_{1} \sigma \quad \phi_{2}=\omega_{2} \tau-v \omega_{2} \sigma
$$

Since we have fixed $\psi=\pi / 4$ the equation of motion for $\psi$ imposes the constraint $\omega_{1}=\omega_{2}=\omega_{12}$ ${ }^{6}$. Plugging this into the Virasoro constraints leads to (we use $y=\sigma-v \tau$ ):

$$
\begin{aligned}
& \partial_{y} g=\frac{v z^{2}}{\left(1-v^{2}\right)\left(1-z^{2}\right)} \\
& \left(z^{\prime}\right)^{2}=\frac{z^{2}}{\left(1-v^{2}\right)^{2}}\left[\left(1-v^{2}\right)\left(1-\omega_{12}^{2}\left(1-v^{2}\right)\right)-\left(1-\omega_{12}^{2}\left(1-v^{2}\right)^{2}\right) z^{2}\right]
\end{aligned}
$$

\footnotetext{
${ }^{5}$ Different multispin string configurations on $A d S_{5} \times S^{5}$ and their connections with known integrable models were studied in [20]. In [11] some revant multi spin rotating string configurations were analyzed.

${ }^{6}$ We thank the authors of [42] for pointing out an error in the first version of this paper.
} 
For future convenience we define:

$$
\xi=\sqrt{\frac{\left(1-v^{2}\right)\left(1-\omega_{12}^{2}\left(1-v^{2}\right)\right)}{1-\omega_{12}^{2}\left(1-v^{2}\right)^{2}}} \quad \gamma=\sqrt{\frac{1-\omega_{12}^{2}\left(1-v^{2}\right)}{1-v^{2}}}
$$

We can integrate the equation for $z(y)$ and find:

$$
z=\frac{\xi}{\cosh (\gamma y)}
$$

Now we present the expressions for the quantities of interest:

$$
\begin{aligned}
& p=\int_{-r}^{r} d \sigma \phi_{3}^{\prime} \quad E=\frac{\sqrt{\lambda}}{2 \pi} \int_{-r}^{r} d \sigma \quad J_{3}=\frac{\sqrt{\lambda}}{2 \pi} \int_{-r}^{r} d \sigma\left(1-z^{2}\right) \dot{\phi}_{3} \\
& J_{1}=\frac{\sqrt{\lambda}}{2 \pi} \int_{-r}^{r} d \sigma \frac{z^{2}}{2} \dot{\phi}_{1} \quad J_{1} \frac{\sqrt{\lambda}}{2 \pi} \int_{-r}^{r} d \sigma \frac{z^{2}}{2} \dot{\phi}_{2}
\end{aligned}
$$

Using the solution we have for $z(y)$ we can solve this integrals and find (we take $r \rightarrow \infty$ ):

$$
\begin{aligned}
& E-J_{3}=\frac{\lambda \xi^{2}}{\pi \gamma\left(1-v^{2}\right)} \quad \xi=\sin \left(\frac{p}{2}\right) \\
& J_{1}=\frac{\lambda \xi^{2}}{2 \pi \gamma} \omega_{12} \quad J_{2}=\frac{\lambda \xi^{2}}{2 \pi \gamma} \omega_{12}
\end{aligned}
$$

Now using the relation:

$$
\omega_{12}^{2}+\frac{\gamma^{2}}{\xi^{2}}=\frac{1}{\left(1-v^{2}\right)^{2}}
$$

We find the dispersion relation for the two spin giant magnon:

$$
E-J_{3}=\sqrt{\left(J_{1}+J_{2}\right)^{2}+\frac{\lambda}{\pi^{2}} \sin ^{2}\left(\frac{p}{2}\right)}
$$

This is a dispersion relation which reproduces the one for giant magnons with one and two spins found in [4] and [5]. We see that the dispersion relation for our simple two spin giant magnon anzatz effectively reduces to the 2 spin dispersion relation (2.27) if we identify $J_{3} \rightarrow$ $J_{1}, J_{1}+J_{2} \rightarrow J_{2}$. From spin chain side one can think of this result as it was discussed in [5] for two spin bound states (see discussion after eq. (16)). Therefore the result can be interpreted 
as superposition of two wavepackets - one in $\varphi_{1}$ another in $\varphi_{2}$ direction. Thus we see that our solution is a particular case of the more general 3 spin giant magnon found in [42]. The solution we found somehow looks similar to the situation in the magnon excitations in the deformed $A d S_{5} \times S^{5}$ considered in [9] - to obtain the single spin magnon dispersion relation one should involve dynamics on another angle from five sphere, while in the limit $\beta \rightarrow 0$ it still reproduces single magnon case.

\section{$3 \quad \beta$-deformed $A d S_{5} \times S^{5}$}

Here we review the $\beta$-deformed $A d S_{5} \times S^{5}$ background found by Lunin and Maldacena [22] This background is conjectured to be dual to marginal deformations of $\mathcal{N}=4 \mathrm{SYM}$. We note that this background can be obtained from pure $A d S_{5} \times S^{5}$ by a series of TsT transformations as described in [23]. Here we will restrict ourselves to the case of real deformation parameter. The resulting supergravity background for string theory dual of real $\beta$-deformations of $\mathcal{N}=4$ SYM is:

$$
d s^{2}=R^{2}\left(d s_{A d S_{5}}^{2}+\sum_{i=1}^{3}\left(d \mu_{i}^{2}+G \mu_{i}^{2} d \phi_{i}^{2}\right)+\tilde{\gamma}^{2} G \mu_{1}^{2} \mu_{2}^{2} \mu_{3}^{2}\left(\sum_{i=1}^{3} d \phi_{i}^{2}\right)\right)
$$

This background includes also a dilaton field as well as RR and NS-NS form fields. The relevant form for our classical string analysis will be the antisymmetric B-field:

$$
B=R^{2} \tilde{\gamma} G\left(\mu_{1}^{2} \mu_{2}^{2} d \phi_{1} d \phi_{2}+\mu_{2}^{2} \mu_{3}^{2} d \phi_{2} d \phi_{3}+\mu_{1}^{2} \mu_{3}^{2} d \phi_{1} d \phi_{3}\right)
$$

In the above formulas we have defined

$$
\begin{aligned}
& \tilde{\gamma}=R^{2} \gamma \quad R^{2}=\sqrt{4 \pi g_{s} N}=\sqrt{\lambda} \\
& G=\frac{1}{1+\tilde{\gamma}^{2}\left(\mu_{1}^{2} \mu_{2}^{2}+\mu_{2}^{2} \mu_{3}^{2}+\mu_{1}^{2} \mu_{3}^{2}\right)} \\
& \mu_{1}=\sin \theta \cos \alpha \quad \mu_{2}=\cos \theta \quad \mu_{3}=\sin \theta \sin \alpha
\end{aligned}
$$

Where $\left(\theta, \alpha, \phi_{1}, \phi_{2}, \phi_{3}\right)$ are the usual $S^{5}$ variables and the deformation parameter is $\beta=\gamma+i \sigma_{d}$ is complex in general, but in our analysis we will consider $\sigma_{d}=0$ 


\subsection{Two spin giant magnons in $\beta$-deformed $A d S_{5} \times S^{5}$}

We will follow [9] and consider semiclassical strings on the $R \times S^{3}$ part of the deformed LuninMaldacena background. The metric and the antisymmetric NS-NS field are:

$$
\begin{gathered}
d s^{2}=-d t^{2}+d \theta^{2}+G \sin ^{2} \theta d \phi_{1}^{2}+G \cos ^{2} \theta d \phi_{2}^{2} \\
B=\tilde{\gamma} G \sin ^{2} \theta \cos ^{2} \theta
\end{gathered}
$$

where

$$
G=\frac{1}{1+\tilde{\gamma}^{2} \sin ^{2} \theta \cos ^{2} \theta}
$$

The Polyakov action takes the form:

$$
\begin{aligned}
& S_{P}=\frac{\sqrt{\lambda}}{4 \pi} \int d \tilde{d} \sigma\left[-\left(\partial_{\tau} t\right)^{2}-\left(\partial_{\tau} \theta\right)^{2}+\left(\partial_{\sigma} \theta\right)^{2}+G \sin ^{2} \theta\left(\left(\partial_{\sigma} \phi_{1}\right)^{2}-\left(\partial_{\tau} \phi_{1}\right)^{2}\right)+\right. \\
& \left.G \cos ^{2} \theta\left(\left(\partial_{\sigma} \phi_{2}\right)^{2}-\left(\partial_{\tau} \phi_{2}\right)^{2}\right)-2 \tilde{\gamma} G \sin ^{2} \theta \cos ^{2} \theta\left(\partial_{\tau} \phi_{2} \partial_{\sigma} \phi_{1}-\partial_{\tau} \phi_{1} \partial_{\sigma} \phi_{2}\right)\right]
\end{aligned}
$$

We define the variable $y=c x-d t$ and require $c^{2}-d^{2}=1$ such that we always have $v=d / c \leq$ 1. Now we will investigate the following rotating string anzatz (which is consistent with the equation of motion and the two Virasoro constraints).

$$
\theta=\theta(y) \quad \phi_{1}=t+g_{1}(y) \quad \phi_{2}=\nu t+g_{2}(y)
$$

This anzatz was studied in [7] in the pure $A d S_{5} \times S^{5}$ background and describes a giant magnon with two spins, one of which is going to infinity and the other is finite. By using the equations of motion derived from the Polyakov action and the two Virasoto constraints we find:

$$
\begin{aligned}
& \partial_{y} g_{1}=\frac{d}{G \sin ^{2} \theta}-d-c \nu \tilde{\gamma} \cos ^{2} \theta \\
& \partial_{y} g_{2}=-\nu d-c \tilde{\gamma} \sin ^{2} \theta \\
& \partial_{y} \theta=\cos \theta \sqrt{1-(\nu c+\tilde{\gamma} d)^{2}-d^{2} \frac{\cos ^{2} \theta}{\sin ^{2} \theta}}
\end{aligned}
$$


We can integrate the last of these equations and find:

$$
\cos \theta=\frac{\cos \theta_{0}}{\cosh \left(\sqrt{1-(\nu c+\tilde{\gamma} d)^{2}} y\right)}
$$

where we have defined

$$
\cos \theta_{0}=\sqrt{\frac{1-(\nu c+\tilde{\gamma} d)^{2}}{c^{2}-(\nu c+\tilde{\gamma} d)^{2}}}
$$

Following [4] and [9] we will identify

$$
\cos \theta_{0}=\sin \left(\frac{p}{2}-\pi \beta\right)
$$

where $p$ is the momentum of the magnon and $\beta$ is the deformation parameter (in our case $\beta$ is real and $\tilde{\gamma}=\sqrt{\lambda} \beta$ ). Now it is easy to find the conserved charges in our problem $\left(J_{1}\right.$ and $J_{2}$ correspond to $\phi_{1}$ and $\phi_{2}$ respectively):

$$
\begin{aligned}
& E=\frac{\sqrt{\lambda}}{\pi} \int_{-\infty}^{\infty} d \sigma \\
& J_{1}=\frac{\sqrt{\lambda}}{\pi} \int_{-\infty}^{\infty} d \sigma G \sin ^{2} \theta\left(\left(1-d \partial_{y} g_{1}\right)-\tilde{\gamma} \cos ^{2} \theta \partial_{y} g_{2}\right) \\
& J_{2}=\frac{\sqrt{\lambda}}{\pi} \int_{-\infty}^{\infty} d \sigma G \cos ^{2} \theta\left(\left(\nu-d \partial_{y} g_{2}\right)+\tilde{\gamma} \sin ^{2} \theta \partial_{y} g_{1}\right)
\end{aligned}
$$

Now using our solution for $\theta(y)$ we find:

$$
E-J_{1}=\frac{\sqrt{\lambda} c}{\pi} \frac{\cos ^{2} \theta_{0}}{\sqrt{1-(\nu c+\tilde{\gamma} d)^{2}}}
$$

and

$$
J_{2}=\frac{\nu c+\tilde{\gamma} d}{c}\left(E-J_{1}\right)
$$

From here we find

$$
\left(E-J_{1}\right)^{2}=J_{2}^{2}+\frac{\lambda}{\pi^{2}} \cos ^{2} \theta_{0}
$$


So finally we find the two spin magnon dispersion relation:

$$
E-J_{1}=\sqrt{J_{2}^{2}+\frac{\lambda}{\pi^{2}} \sin ^{2}\left(\frac{p}{2}-\pi \beta\right)}
$$

We note that when the deformation parameter $\beta$ vanishes we reproduce the dispersion relation (2.22) for the two spin magnon in pure $A d S_{5} \times S^{5}$ found. The above result also agrees with the conclusions of [28] that in the deformed theory we should modify the asymptotic Bethe ansatz equations by the transformation $p \rightarrow p-2 \pi \beta$. As expected from the Hofman-Maldacena interpretation ${ }^{7}$ the dispersion relation is periodic in $p$ with period $2 \pi$ and in $\beta$ with period 1 .

\section{Conclusions and Outlook}

In this short paper we investigated the multispin string solutions corresponding to giant magnon excitations. First we give a simple generalization of the two spin string solutions corresponding to magnon excitations. Our solution with two spins in the spherical part is the same as the giant magnons with two spins but in our case string dynamics on another isometry of the sphere is alowed. Although the solution is similar, it extends the slass of string solutions corresponding to two spin magnon excitations. As a second example we considered the giant magnon solution with two spins in the $\beta$-deformed $A d S_{5} \times S^{5}$ background. Our solution looks identical to the one found in [9], but in our case the solution is "essentially" with two spins in the following sense. While in [9] the solution reproduces the one spin solution in the limit $\gamma \rightarrow 0$, in our case this limit reproduces the two spin $A d S_{5} \times S^{5}$ solution obtained in [7], [8].

In conclusion, the Hofman-Maldacena solutions certainly deserves more detailed investigations. One direction for further study is to include more spins from the AdS part of the background, by considering classical strings moving on $A d S_{3} \times S^{3}$ and $A d S_{3} \times S^{5}$ and thus generalizing the solution found in [8] for strings moving on $A d S_{3} \times S^{1}$. It would be interesting also to find the corresponding spin chain dispersion relations. Another possibly interesting problem would be to see how the giant magnon solutions look like in the non-supersymmetric multiparameter $\beta_{i}$-deformed background found by Frolov [23] and further studied in [25],[32],[35]. Some of these issues are currently under investigation.

\footnotetext{
${ }^{7}$ We thank S. Pal for some comments on this point
} 
Note:

After the completion of this paper two interesting papers [41],[42] discussing multispin giant magnons appeared. The authors of [41] analyze different scattering and bound states of one and two spin giant magnons using a technique based on the dressing method for the $S O(N)$ vector model, in the last section of this paper they present a particular three spin solution. It is interesting that the dressing method allows more general time dependence beyond particular ansatz ${ }^{8}$. In [42] a three spin giant magnon solution was found by reductions to Neumann-Rosochatius integrable system. Along with other giant magnon solutions and a thorough analysis on the spin chain side is given.

\section{Acknowledgements}

We thank M. Kruczenski, J. Russo and A.A. Tseytlin for pointing out a mistake in the first version of our paper. We thank A.A. Tseytlin for usefull comments. The work of N.P.B. is supported by a University of Southern California Graduate Research Fellowship. The work of R.R is partially supported by a Bulgarian NSF CNVUY-10/66 grant.

\footnotetext{
${ }^{8}$ We thank M. Spradlin for pointing this to us.
} 


\section{References}

[1] J.M. Maldacena, "The Large $\mathcal{N}$ Limit of Superconformal Field Theories and Supergravity", Adv.Theor.Math.Phys. 2 (1998) 231-252; Int.J.Theor.Phys. 38 (1999) 1113-1133, hep-th/ 9711200; E. Witten, "Anti-de Sitter space and holography", Adv. Theor. Math. Phys. 2, 253 (1998), hep-th/9802150; S. S. Gubser, I. R. Klebanov and A. M. Polyakov, "Gauge theory correlators from non-critical string theory", Phys. Lett. B 428 , 105 (1998), hepth/9802109.

[2] D. Berenstein, J. M. Maldacena and H. Nastase, "Strings in flat space and pp waves from $\mathcal{N}=4$ Super Yang Mills", JHEP 0204, 013 (2002), hep-th/0202021.

[3] S. S. Gubser, I. R. Klebanov, A. M. Polyakov "A semi-classical limit of the gauge/string correspondence", Nucl.Phys. B 636 (2002) 99-114, hep-th/0204051

[4] Diego M. Hofman, Juan Maldacena "Giant Magnons", hep-th/0604135

[5] Nick Dorey "Magnon Bound States and the AdS/CFT Correspondence", hep-th/0604175

[6] Heng-Yu Chen, Nick Dorey, Keisuke Okamura "Dyonic Giant Magnons", hep-th/0605155

[7] Gleb Arutyunov, Sergey Frolov, Marija Zamaklar, "Finite-size Effects from Giant Magnons", hep-th/0606126

[8] J.A. Minahan, A. Tirziu, A.A. Tseytlin "Infinite spin limit of semiclassical string states", hep-th/0606145

[9] Chong-Sun Chu, George Georgiou, Valentin V. Khoze Magnons "Classical Strings and beta-Deformations", hep-th/0606220

[10] N. Beisert "The su(2|2) Dynamic S-Matrix", hep-th/0511082

[11] Shijong Ryang, "Wound and Rotating Strings in $A d S_{5} \times S^{5}$ ", JHEP 0508 (2005) 047; hep-th/0503239

[12] J. A. Minahan and K. Zarembo, "The Bethe-ansatz for $\mathcal{N}=4$ super Yang-Mills", JHEP 0303, 013 (2003), hep-th/0212208.

[13] N. Beisert, "The dilatation operator of $\mathcal{N}=4$ super Yang-Mills theory and integrability", Phys. Rept. 405, 1 (2005), hep-th/0407277. 
[14] A. A. Tseytlin "Spinning strings and AdS/CFT duality", hep-th/0311139; A. A. Tseytlin "Semiclassical Strings and AdS/CFT", hep-th/0409296

[15] J. Plefka, "Spinning strings and integrable spin chains in the AdS/CFT correspondence", hep-th/0507136.

[16] M. Kruczenski, "Spin chains and string theory", Phys.Rev.Lett. 93 (2004) 161602, hepth/0311203.

[17] H. Dimov and R.C. Rashkov "A note on spin chain/string duality", Int.J.Mod.Phys. A20 (2005) 4337, hep-th/0403121.

[18] Rafael Hernandez, Esperanza Lopez, "The SU(3) spin chain sigma model and string theory", JHEP 0404 (2004) 052, hep-th/0403139.

[19] B. Stefanski, jr., A.A. Tseytlin, "Large spin limits of AdS/CFT and generalized LandauLifshitz equations", JHEP 0405 (2004) 042, hep-th/0404133; B. Stefanski, jr., A.A. Tseytlin, "Super spin chain coherent state actions and $A d S_{5} \times S^{5}$ superstring", Nucl.Phys. B718 (2005) 83-112, hep-th/0503185.

[20] G. Arutyunov, J. Russo and A. A. Tseytlin, "Spinning strings in AdS $\times S^{5}:$ New integrable system relations", Phys. Rev. D 69, 086009 (2004), hep-th/0311004; G. Arutyunov, S. Frolov, J. Russo and A. A. Tseytlin, "Spinning strings in $A d S_{5} \times S^{5}$ and integrable systems", Nucl. Phys. B 671, 3 (2003), hep-th/0307191.

[21] I. Bena, J. Polchinski and R. Roiban "Hidden symmetries of the $A d S_{5} \times S^{5}$ superstring"; Phys. Rev. D 69 (2004) 046002; hep-th/0305116

[22] O. Lunin, J. Maldacena "Deforming field theories with $U(1) \times U(1)$ global symmetry and their gravity duals", JHEP 0505 (2005) 033, hep-th/0502086

[23] Sergey Frolov "Lax Pair for Strings in Lunin-Maldacena Background", JHEP 0505 (2005) 069, hep-th/0503201

[24] S. Frolov, R. Roiban, A. Tseytlin, "Gauge-string duality for superconformal deformations of $\mathcal{N}=4$ Super Yang-Mills theory", JHEP 0507, 045 (2005), hep-th/0503192.

[25] S. A. Frolov, R. Roiban and A. A. Tseytlin, "Gauge-string duality for (non)supersymmetric deformations of $\mathrm{N}=4$ super Yang-Mills theory", Nucl. Phys. B 731, 1 (2005), hepth/0507021. 
[26] N. P. Bobev, H. Dimov and R. C. Rashkov, "Semiclassical strings in Lunin- Maldacena background", hep-th/0506063.

[27] S. Ryang, Rotating strings with two unequal spins in Lunin-Maldacena background", JHEP 0511, 006 (2005), hep-th/0509195.

[28] N. Beisert and R. Roiban, "Beauty and the twist: The Bethe ansatz for twisted $N=4$ SYM", JHEP 0508, 039 (2005), hep-th/0505187.

[29] R. de Mello Koch, J. Murugan, J. Smolic and M. Smolic, "Deformed PP-waves from the Lunin-Maldacena background", JHEP 0508, 072 (2005), hep-th/0505227

[30] T. Mateos, "Marginal deformation of $N=4 S Y M$ and Penrose limits with continuum spectrum", JHEP 0508, 026 (2005), hep-th/0505243

[31] R. de Mello Koch, N. Ives, J. Smolic and M. Smolic, "Unstable giants", Phys. Rev. D 73, 064007 (2006), hep-th/0509007

[32] A. H. Prinsloo, "gamma(i) deformed Lax pair for rotating strings in the fast motion limit", JHEP 0601, 050 (2006), hep-th/0510095

[33] H. Y. Chen and S. Prem Kumar, "Precision test of AdS/CFT in Lunin-Maldacena background", JHEP 0603, 051 (2006), hep-th/0511164

[34] W. H. Huang, "Semiclassical rotating strings in electric and magnetic fields deformed AdS(5) x $S^{* * 5}$ spacetimes", Phys. Rev. D 73, 026007 (2006), hep-th/0512117

[35] L. F. Alday, G. Arutyunov and S. Frolov, "Green-Schwarz strings in TsT-transformed backgrounds", hep-th/0512253

[36] H. Y. Chen and K. Okamura, "The anatomy of gauge / string duality in Lunin-Maldacena background", JHEP 0602, 054 (2006), hep-th/0601109

[37] T. McLoughlin and I. Swanson, "Integrable twists in AdS/CFT", hep-th/0605018

[38] U. Gursoy and C. Nunez, "Dipole deformations of $N=1$ SYM and supergravity backgrounds with U(1) x U(1) global symmetry", Nucl. Phys. B 725, 45 (2005), hep-th/0505100

[39] N.P. Bobev, H. Dimov and R.C. Rashkov "Semiclassical Strings, Dipole Deformations of $\mathcal{N}=1$ SYM and Decoupling of KK Modes", JHEP 0602 (2006) 064, hep-th/0511216 
[40] U. Gursoy, "Probing universality in the gravity duals of $N=1 S Y M$ by gamma deformations", JHEP 0605, 014 (2006), hep-th/0602215.

[41] Marcus Spradlin, Anastasia Volovich, "Dressing the Giant Magnon", hep-th/0607009

[42] M. Kruczenski, J. Russo, A.A. Tseytlin, "Spiky strings and giant magnons on $S^{5}$ ", hepth/0607044 